\title{
CONFERÊNCIAS
}

\section{LES POLITIQUES DE L'ARCHIVE VIVE}

\section{SAM BOURCIER ${ }^{1}$}

\section{Bom dia todxs}

Un grand merci pour l'invitation.

Se revoir bientôt

Mot sur l'écriture inclusive

La question que j'aimerais traiter aujourd'hui, c'est celle de la politique des archives. Pourquoi les/nos archives, c'est politique ? Comment nous pouvons en faire et en faire un instrument de lutte, de résistance et de subjectivation à l'époque néolibérale autoritaire, à l'époque du capitalisme numérique et de la bio-archive ? C'est toute la question ou les questions à laquelle répond l'archive vive, l'archive kuir et transféministe. Et pour en parler je vais partir comme je le fais tjs d'une expérience personnelle \& politique : c'est-à-dire mon engagement depuis plus de 20 ans pour la création d'un centre d'archives LGBTQI à Paris. Ce serait trop long et pas intéressant de vous raconter ce feuilleton pas très ragoutant pour la mairie PS (Parti Socialiste) de Paris mais qui est très instructif pour comprendre ce que sont les centres d'archives LGBTQI autonomes et communautaires et ce que font les capitales mondiales aux cultures LGBTQI, comment elles sont devenues des entreprises de mémoire LGBTQI. Et pas pour le meilleur.

Pour faire vite, il se trouve que la mobilisation pour la création de ce centre d'archives a repris en 2017 à l'initiative d'Act-Up avec la création du Collectif Archives LGBTQI et que nous venons de gagner cette bataille avec un vote unanime du conseil de

\footnotetext{
${ }^{1}$ Université de Lille Collectif Archives LGTQI
}

Vol. 03, N. 11, Jul. - Set., 2020 - http://periodicoscientificos.ufmt.br/ojs/index.php/rebeh/index 
Paris en février dernier. Ce vote acte notre projet de centre qui est communautaire et autonome : 1/communautaire, ça veut dire que le centre sera un centre culturel géré par et pour la communauté LGBTQI ; Autonome, ça veut dire que la mairie ou les archives nationales ne pourront pas contrôler notre politique scientifique et culturelle ou notre philosophie de l'archive. Elles ne pourront pas non plus interférer avec notre gouvernance ou faire du centre un instrument de pinkwashing et d'extractivisme au service du tourisme LG (lesbien et gay) global et de «Paris Capitale de l'amour». A cet égard, la politique des plaques de rues \& jardins parisienne pour les femmes est significative. La mairie inaugure un jardinet Marielle Franco en 2019 après avoir déroulé pendant des mois son portrait sur la façade de la mairie centrale, les relations internationales de la Mairie de Paris obligent le Collectif Archives LGBTQI à mettre un portrait de Marielle Franco crêpé de noir sur la scène au cours d'une journée archives organisée par le collectif en mai 2018 avec une biographie qui ne mentionne pas le fait qu'elle était une militante lesbienne mais quand on leur demande une aide à la traduction pour son livre, UPP, il n'y a plus personne et pas d'argent.

Ce que je retire de cette longue lutte collective, c'est que pour faire ce que nous avons à faire, pour ne pas se laisser déposséder de notre force archivale, qui est réelle et originale, culturelle et épistémopolitique, généreuse, intelligente et juste, véritablement démocratique, notre regard sur les archives doit changer. Il doit devenir politique. Il faut aussi changer le regard que portent les archives institutionnelles sur nous, s'opposer à la violence archivale qu'elles génèrent, les obliger à nous respecter et aussi faire sans elles.

La $1^{\text {ère }}$ chose à faire peut-être pour changer notre regard sur l'archive, c'est de réaliser sa dimension biopolitique et de la voir comme un continuum. Si on ne part pas de l'omniprésence de l'archive dans nos vies, on ne comprend pas son ampleur, le découpage de son flux, la volonté de contrôle qu'elle nourrit et sa fibre biopolitique.

Car nous sommes des archivés, tout le temps, toute notre vie et dès les premiers jours. Notre première archivation, c'est la déclaration performative à l'état civil : le «c'est une fille », «c'est un garçon » ou « autre chose »... Et puis, on se fait archiver par l'administration, les institutions médicales, militaires, policières et juridiques. Et puis, on sera travaillé en tant qu'archives par les chercheurs. La fonction administrative de 


\section{员드댄}

l'archive est première mais nous l'oublions. Il faut dire que tout est fait pour que notre vision de l'archive se réduise à celle d'un reste poussiéreux ou précieux, bien gardé quelque part et plutôt réservé aux historiens qui sauraient quoi en faire pour notre plus grand bien. Or il ne faut pas réserver l'archive aux historiens ou au passé. L'archive ne peut pas être prisonnière de l'histoire, d'une seule discipline et a fortiori de l'écriture, de l'écriture de l'histoire. J'y reviendrai.

L'archive, c'est donc une machine. C'est les archives au travail avant qu'elles ne soient déposée dans les centres archives institutionnels : comme les archives nationales en France fondées au XVIIIème siècle dans le sillage de la Révolution Française, ou les archives nationales du Brésil fondées au XIXème siècle. Il existe une relation archivale circulaire entre l'état centralisateur ou fédéral et ses administrations: l'armée, les colonies, la police, la justice, etc. Elles carburent à l'archive parce qu'elles gouvernent grâce à elles. L'archivation est donc d'abord un principe de production et de gestion de l'archive à des fins d'organisation et de contrôle. Cette archive administrative, bureaucratique, massive, énorme, active $\mathrm{H} 24$, cette archivadministration est à l'opposé de la représentation courante qui fait de l'archive quelque chose de résiduel, d'incomplet, d'inerte que viendrait ressusciter l'historien qui va consulter dans un centre d'archive. C'est vrai que l'argument temporel de l'archive des historiens, naturalisant et biologisant, est puissant : les gens vivent et laissent des archives après leur mort. L'archive, c'est du passé, du post-mortem pour les corps qui les ont produites. Au point de faire oublier que les archives travaillent et gouvernent les corps vivants. Les archives, c'est d'abord des rapports de pouvoir. C'est une machine épistémologique qui carbure aux savoirs. L'état, les institutions ne peuvent exercer leur pouvoir sans tous les savoirs que génère leur masse archivale.

Les archivadministrations ne produisent donc pas que le citoyen et l'historien. Il faut y ajouter la production et le contrôle des populations, des subjectivités, des corps et des identités nationalisées et subalternisées. On pense évidemment à la scienta sexualis, à la scienta racialis et aux administrations coloniales du XIXème. Voilà le bouillonnement que l'on voit quand on se place au tout début de la chaîne archivale. 


\section{员드댄}

Archiver c'est gouverner, y compris en contrôlant notre désir d'archive et c'est bien ce qu'on nous a fait comprendre au cours de ces 3 dernières années de luttes pour le centre d'archives.

Nous avons ressenti très concrètement ce que Derrida a appelé « la violence des archontes » dans son fameux Mal d'archive paru dans les années 90. Les archontes étaient les magistrats grecs qui gardaient et faisaient l'archive administrative et juridique chez eux, depuis leur maison. Pour Derrida, ils sont, je cite «l'illusion du commencement et l'arrivée du commandement » et j'ajoute dans un bâtiment. Dès que se produit ce que Derrida appelle «rassemblement », c'est-à-dire ce regroupement des archives dans un lieu institutionnel dédié, cette phase de concentration matérielle va déclencher sur fond de complicité intense avec l'état, des suppressions d'archives, des dépossessions, des restrictions, des codifications d'accès, des sélections au profit des documents écrits, des typologisations : par exemple les minorités sexuelles et de genre sont généralement des archives dites « sensibles » parce que médicales ou politiques.

Mais ce rassemblement se traduit surtout par une séparation d'avec les corps qui les produisent, qui peuvent les utiliser ou les remettre en circulation de leur vivant, une séparation des supports (on va distinguer entre les documents, les livres et les objets), la séparation entre les producteurs.trices d'archives et les archivistes en charge de l'inventaire, de l'indexation et des descriptions. Et si vous demandez un lieu autonome pour faire ça, on vous le refuse. En tout cas en France, au motif que vous devez intégrer les archives nationales et que vous y serez les bienvenus et bien traités, ce qui est structurellement et politiquement faux évidemment. Pour toute une série de raisons sur lesquelles on pourra revenir.

Le principe archontique est donc un principe de séparation, de dissociation précisément parce que les archives recèlent un pouvoir de rassemblement des personnes et des corps dans une temporalité qui n'est pas celle du passé mais du présent et du futur antérieur. L'archive est une force collective et politique. C'est cette force de l'archive qui est capturée et réservée. 


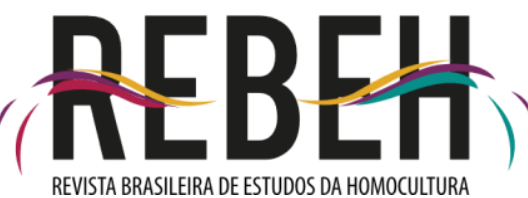

Mais on peut s'opposer à la violence archivale des archontes et à la violence administrative des archives. On peut briser nos chaînes archivales. Et c'est la $2^{\text {ème }}$ chose importante à faire pour changer notre regard et nos usages de l'archive. Comment? En tirant parti de ce que j'appelle l'itérabilité de l'archive.

L'itérabilité de l'archive, c'est tout ce qui relève de sa production, de sa reproduction, de sa répétition, de sa réactivation et de sa circulation et singulièrement quand elle est le fait d'autres acteurs que les acteurs traditionnels de l'archive. Le fait des archivacteurs.trices archi-vivant.e.s

L'itérabilité c'est est à la fois l'iteratio (la répétition) et l'itinerarius (la voyage) de l'archive. Elle est aussi assurée et comment! par les minoritaires et leurs centres d'archives communautaires dont le premier des soucis est justement de ne pas séparer ou de ne pas disloquer mais de faire voir et de faire re-circuler les archives. Ils multiplient les boucles d'archives, les circuits courts en somme, là où les institutions transforment les archives en chaînes et multiplient les obstacles à leur consultation et leur dissémination. C'est cette itérabilité qui est bridée par les garde-chiourmes des archives dont font partie les gatekeepers des sciences sociales. Car il n'y a pas que les historiens qui ont mis le grappin sur les archives ! L'itérabilité de l'archive, c'est son potentiel de non séparation des corps producteurs.trices d'archives d'avec les archives et leur pouvoir rassembleur, collectif et politique dans une temporalité et des projets communs. Les archontes ne peuvent pas rivaliser avec un site, un podcast, un groupe facebook, une performance, des histoires orales sans fin, une cartographie, un atelier d'archives, fabriqués par les archivés devenus archivacteurs.archivactrices, foyers d'archives plus que sources d'archives. Bref, il est possible et nécessaire d'opposer l'itérabilité de l'archive au principe, à la violence archontique.

La $3^{\text {ème }}$ chose pour voir et faire les archives autrement, c'est de réaliser que la grande force de l'archive vive, c'est sa dimension performative et à beaucoup de niveaux.

Les archivistes professionnels -enfin ceux qui sont passés à côté du tournant archival des années 90- prétendent organiser les restes et les traces a posteriori en toute objectivité. Ils nient la dimension performative de l'archive au sens où il y a toujours quelqu'un ou quelque chose qui décide de la découpe de l'archive. Son incomplétude 
n'est pas naturelle. Cette incomplétude, les minoritaires en sont très conscients. Elle est grande et souvent cruelle mais elle nous appartient. L'archive est toujours incomplète mais cette incomplétude prend des formes spécifiques pour les minoritaires dont font partie les LGBTQI. Ils ont été à la fois exclus des archives officielles et de la culture en général mais aussi maltraités et mal représentés par elles. La censure à leur égard ne s'est pas exercée de manière simplement négative, par effacement mais aussi de manière positive. Tout le XIXème siècle et une bonne partie du XXème a visibilisé-créé-archivé les invertis, les homosexuels, les racisés, les débiles, les indigents, les indigènes, les misérables, les identités sexuelles et racisées en les médicalisant et en les criminalisant. Cette histoire de la violence se confond avec la grande archive du sexe et de la race.

Il est difficile et souvent sisyphéen d'archiver les trous de l'histoire et les silences de la représentation, même si c'est un réflexe compréhensible. C'est tout le problème de l'archive des minorités, des sans voix et des subalternes. Les trous sont tellement béants qu'on a pu y répondre par la fiction, ce qui nous rappelle aussi la dimension performative de l'archive et l'éloigne du régime de vérité auquel prétendent les historiens. C'est ce que dit Wittig dans Les Guerillères pour contourner l'effacement des lesbiennes et des femmes dans la mémoire : «Tu dis qu'il n’y a pas de mots pour décrire ce temps, tu dis qu'il n'existe pas. Mais souviens-toi. Fais un effort pour te souvenir. Ou, à défaut, inventes ». On retrouve la même stratégie fictionnelle avec le film The Watermelon Woman de Cheryl Dyune sorti en 1996. La réalisatrice noire lesbienne du film y raconte l'histoire d'une actrice noire lesbienne à Hollywood dans les années 30. À la fin du film, qui joue sur les codes réalistes du documentaire, on comprend que les archives et les photos qu'on y voit ont toutes été inventées. Le film illustre l'impossible restitution et l'effacement des femmes noires dans le cinéma mais aussi les vertus de la fiction performative plus que corrective ou réparatrice.

L'autre niveau performatif est celui qui consiste à réintroduire massivement l'archive orale dans la production des archives des minoritaires. C'est toujours la parente faible des archives et de l'histoire, cette archive orale... Mais il ne s'agit pas d'opposer écriture et oralité. Il s'agit de distinguer des modes de production et de diffusion de l'archive très différents et de voir lequel est archi dominant. Le dispositif d'histoire- 


\section{R드댄}

écriture est saturé de colonialité. Il s'est construit contre l'oralité et au détriment de ce que Diana Taylor appelle l'archive-répertoire. L'archive-répertoire ne supporte pas la séparation entre l'écrit, l'oral et le corps. On y trouve des rituels, des événements, des performances au sens actuel du terme, des gestes, des choses immatérielles, des textes et des inscriptions. Il n'y a pas que des formes orales. Et c'est archivable comme l'a bien compris l'Unesco qui a entreprit de sauver-archiver le patrimoine mondial immatériel. La performance au sens très large du terme est un médium de transmission de la mémoire et de la culture. La dictature de l'écriture et du texte sert précisément la disqualification de la transmission archivale performative. La force de l'itérabilité de cette archive contraste avec le système de transfert bloqué, grippé, voire élitiste qui est celui de l'archive-histoire, de l'archive-écriture, des archives décorporalisées et décollectivisées. La performance est une force de transmission et de synchronisation puisqu'elle rassemble les corps dans un temps commun voire un espace commun.

La solution culturelle communautaire est donc de permettre aux personnes concernées d'assurer la conservation et la transmission de ce type d'archive et de ce mode de transmission. De manière collatérale, la question devient : comment culturaliser l'archive en la retirant des griffes de l'histoire ? Cela revient à dire qu'il faut rompre avec l'approche mono-disciplinaire de l'archive qui en fait le terrain de jeu de l'histoire ou des sciences sociales comme l'anthropologie, l'ethnologie ou la sociologie.

Cette tension entre histoire et cultures, les minoritaires et les subcultureux la connaissent bien, parce qu'iels ne courent pas tant après leur «patrimoine » ou leur « matrimoine », qu'après leur répertoire, leurs archives-outils, des moyens de faire vivre par l'archive, leurs cultures, leurs subjectivités, des formes de vie et leurs luttes parce qu'elles proposent une vision critique et différente du monde qui leur est nécessaire. La performance au sens actuel du terme dont on sait la proximité qu'elle entretient avec l'archive-répertoire est donc l'une des ressources majeures de cette archive vive qu'ils partagent. Et je vais en donner un exemple avec la performance de Juan da Silva et de Koriangelis Brawns au festival des putes du SNAP (S.ex workers N.arratives A.rts \& P.olitics). 


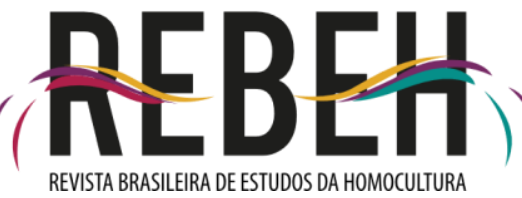

Le 3 novembre 2018, j'ai donc vu la performance de Juan et de Kori, à une soirée du festival. C'est là que j'ai appris que Juan était aussi Marica, là en drag. En l'interviewant le lendemain pour le premier recueil d'archives orales que réalise le collectif Archives LGBTQI+, j'ai appris que Marica faisait partie d'un collectif en Colombie, les Miau qui réalisait des performances, des vidéos U tube et qui organisait des défilés et des concours de beauté trans. C'était avant que Juan ne quitte Bogota, vu que s'il était resté en Colombie, il serait probablement mort assassiné. Pour avoir été un activiste LGBT visible et vocal ou pour ne pas avoir bénéficié des derniers traitements pour le VIH. Tout ça, je l'ai aussi appris, je l'ai vu, en regardant les images et les soustitres qui défilaient sur l'écran de la scène du festival. Ils se/nous racontaient leur vie, pendant que Kori maquillait Marica. Ce carambolage entre auto-récit, performance et histoire orale m'a amené à m'interroger sur la relation entre performance, histoire orale et auto-archive ? Comment recircule plutôt que «s'écrit » 1'histoire collective avec la performance?

Avec la performance au SNAP, l'histoire de Juan et de Kori a été publicisée. Nous avons assisté à la création d'une mémoire publique. La performance sur scène produit plutôt qu'elle ne «garde » la mémoire ou une archive en proposant une forme de remémoration active et engagée. Ca vaut aussi pour tout recueil d'histoires orales, s'il n'est pas réduit à une collection d'histoires ou d'informations. Et là, tout dépend des politiques du savoir pratiquées. D'un point de vue épistémopolitique, on sait qu'il y a des disciplines attitrées pour fondre sur les archives ou faire de l'histoire orale. L'histoire, l'anthropologie ou l'ethnologie par exemple. L'histoire voit majoritairement l'interview comme de l'information à évaluer, à recouper avec d'autres (c'est son côté flic scientifique), ou encore comme une preuve, une illustration de la thèse ou de la chronologie que concocte souvent seul l'historien. Mais l'on peut aussi considérer les histoires de vie, les entretiens comme des savoirs à diffuser, des savoirs productifs et performatifs. C'est d'ailleurs ce qui sépare les anthropologues et les historiens classiques et les producteurs d'archives féministes, queer, transféministes et communautaires. Iels n'ont pas les mêmes politiques et la même éthique de l'entretien. Iels n'ont pas la même vision des rapports de savoir/pouvoir que génère le fait de faire des entretiens. Iels 


\section{员드댄}

considèrent le récit, l'histoire de vie comme une performance et ils développent des dispositifs performatifs de génération et de circulation des archives. Iels tiennent compte du corps, de l'intonation, des gestes, des mimiques, de la langue, de tout ce qui fait qu'un entretien est une performance incarnée, de tout ce qui disparaît généralement dans la transcription de l'interview qui réduit la personne et l'entretien à un texte.

Ces usages de la performance sont différents du reenactement d'histoires de vie ou d'événement comme outil de valorisation des archives dans les musées qui ont connu un boom depuis le tournant archival des années 80-90. Le tournant archival recouvre essentiellement l'utilisation des archives dans l'art et l'utilisation de métaphores ou de concepts qui relèvent de l'archivistique dans les pratiques artistiques. Ici, il s'agit de spectacles de performances à partir d'histoires ou de récits de vie qui relèvent de l'autoarchive comme la performance de Juan et de Kori et du dispositif plus général d'un festival. Ces pratiques performatives relèvent de ce que Della Pollock appelle le remembering comme processus actif plutôt que de la mémoire: «Ce dont il s'agit, c'est de la traduction d'événements subjectivement remémorés en actes de mémoire incarnés, de faire passer la mémoire dans la remémoration (...) C'est ce que fait la représentation de la mémoire vivante, ça fait des choses". Le remembering est un processus incarné, performatif et collectif. L'archive performative permet une production de l'archive en boucles successives et la création de circuits. Elle permet de ne pas se contenter de la chaîne de l'archive traditionnelle qui est celles des archives institutionnelles mais aussi celle des sciences humaines. Dans cette économie des archives souvent élitiste, les chercheur.e.s constituent des réservoirs d'archives orales qui meurent une fois leur "terrain" terminé, vu que les protocoles de consentement n'ont pas prévu en amont d'utilisation archivale ultérieure plus large ou autre que « scientifique ». Les premier.e.s dépossédé.e.s de cette richesse sont les interviewé.e.s. C'est la raison pour laquelle la réactivation des terrains des chercheurs en sciences sociales et une nouvelle éthique de l'entretien sont des objectifs importants pour les praticiens de l'archive vive.

Les dispositifs performatifs de l'archive vive contrecarrent ces phénomènes de dépossession. C'est le cas par exemple avec Like a Family, le spectacle en 1988 de Della Pollock sur l'histoire des cueilleurs de coton en Caroline du Nord basé sur des histoires 


\section{REBE员}

orales. Le spectacle a tourné dans les villes où avaient vécu, ou vivaient les interviewé.e.s. Il a déclenché du retelling avec le public et les habitants, d'autres histoires et d'autres spectacles. C'est aussi le cas avec le projet Sexshunned, un projet collaboratif de Ourstory Scotland basé sur un recueil de récits de vie et d'archives orales LGBT qui ont été montés en spectacle avec le théâtre 7.84. Le projet a généré des ateliers performance et théâtre pour travailler collectivement sur les témoignages oraux à incorporer dans le spectacle.

On voit donc qu'un entretien pour l'histoire orale n'est pas destiné à finir comme une archive dans un centre d'archives, sur un site ou dans une thèse. On mesure la vitalité de l'archive à sa capacité à sortir des placards, des greniers, des boxes, à générer d'autres collectes, d'autres foyers d'archives et non simplement à être mise à disposition dans les institutions. L'entretien d'archive orale, les récits oraux sont aussi faits pour going public dans une performance collective qui se déroule en public, qui publicise les entretiens, les histoires de vie et en suscite d'autres. Qui permet une appropriation de la production des archives. Moyennant quoi, les usages traditionnels de l'archive qui consacrent la reprivatisation ou la confiscation pour un usage élitiste, scientifique ou réservé ne peuvent pas être les seuls. Cette déprivatisation, cette mise en commun que brident les archives des archontes, cette déplacardisation dés-individualise la mémoire et la communalise. Elle la met à disposition et la diffuse. La performance exhibe et diffuse un dispositif, une force archivale communicative qui peut être facilement reproduite et générer autant de pratiques archivales. Cela va contre la dépolitisation des archives que provoque l'individualisation de la mémoire ou sa transformation en une injonction au « devoir de mémoire ». La proximité de ce modèle d'auto-archive collective facilement reproductible entretient une proximité évidente avec les revendications épistémologiques féministes et autonomes des années 70 et + : l'auto-enquête, le DIY, l'autonomie et l'ancrage dans l'expérience. Ce n'est d'ailleurs pas un hasard si on assiste à une prolifération de la forme atelier dans le champ de l'archive queer et transféministe. Les «apéros cartons » organisés par Mémoire des Sexualités à Marseille en sont un bon exemple. Ce n'est pas un hasard non plus si les recueil d'archives orales du Collectif Archives LGBTQI finissent en podcast (40.000 écoutes). 


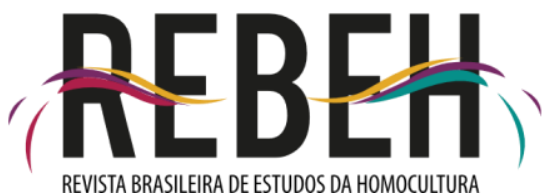

Pour finir, j'aimerais revenir en vitesse faute de temps sur la bio-archive numérique que j'évoquais pour commencer. Elle est une déclinaison de la bio-archive, de la dimension biopolitique de l'archive et je crois que c'est pas mal d'en parler parce que le numérique agit un peu comme une solution magique dès qu'on parle d'archives. On va tout numériser donc tout garder et il suffirait de tout mettre sur le net... Les choses ne sont pas si simples. Mettre les archives sur internet, ça coûte cher et sans design numérique et sans indexation communautaire, ça ne sert pas à grand-chose et ça ne permet pas de trouver ce qu'on cherche à des fins de recherche scientifique comme on dit mais aussi et surtout personnelle et politique pour se construire et agir en tant que LGBTQI dans le monde. En un mot, à avoir recours à l'archive-outil.

Je n'ai pas le temps de le faire ici et c'est un truc que je vais faire dans mon bouquin sur les archives mais il faudrait revenir sur la richesse des pratiques de l'archive et les renommer. A côté de l'archive-histoire sur son piédestal et de l'archive savoir/pouvoir, il y a l'archive-recherche au sens académique du terme. Ces pratiques d'archives se tiennent pas loin de l'archive-preuve de l'état, des juges et des flics. Mais il y a aussi l'archive-culture dont j'ai un peu parlé et l'archive-lutte et cette archiveRecherche justement avec un grand $\mathrm{R}$ cette fois. Celle qui permet la subjectivation personnelle et politique. Ces pratiques d'archives là, moins connues, jamais célébrées, produisent et transmettent aussi des savoirs mais ils n'ont pas la même fonction que les savoirs-pouvoirs. L'archive-lutte et l'archive-recherche avec un grand R sont le réservoir des luttes et des subjectivations politiques. Elles permettent notamment la transmission des scénarios et des cultures militantes dans le temps présent. (ex Act Up).

Pour ce qui est de la bio-archive numérique, c'est celle que nous produisons constamment sur le net, sur les réseaux sociaux en laissant des traces numériques qui sont exploitées par les GAFA. C'est nous cette fois qui générons constamment notre contrôle et notre architecture panoptique avec notre corps et notre espace domestique connectés. Le panopticon de Bentham était le modèle de surveillance et de régulation de la société capitaliste moderne. L'archive native, la cyberarchive virtuelle, est le modèle de contrôle de la société du capitalisme néolibéral numérique actuel. 


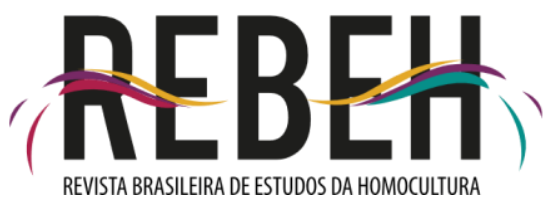

La bio-archive numérique est aussi une auto-archive... mais l'auto-archive du capitalisme numérique est un dévoiement de l'auto-archive autonome et communautaire que pratiquent les minoritaires dans les lieux d'archive qu'iels créent. Et cette machine archive-là, ce que j'appelle le blob de l'archive bio-numérique qui va me produire comme sujet consommateur et citoyen votant pour Trump, le Brexit ou Bolsonaro a bien des points communs avec la machine de l'administration coloniale, de l'archive du sexe et de la race.

Le blob de la bio archive numérique est une réalisation perverse et totale de la pratique archivale de base : la recherche dans les deux acceptions que je viens de donner à ce terme, la Recherche avec un grand $\mathrm{R}$ et la recherche. Avec la capture unilatérale de nos données par les GAFA, le chercheur à tous les sens du terme d'archives à tous les sens du terme, devint matrice archivale à son insu de son plein gré, constamment aspiré par le blob du capitalisme numérique néo-libéral dont le but est d'exploiter la valeur comportementale déduite de nos traces numériques résiduelles : ce que l'on est, traduit par ce qu'on like, ce qu'on cherche, ce qu'on achète, comment on bouge, comment on baise, comment on respire, etc. Le blob de la bio-archive numérique relève du même type d'espace épistémopolitique que celui de l'archive avant l'archive placée en institution : la machine archive dont je parlais pour commencer où usinent les flux et les savoirspouvoirs. Comme la machine archive, le but du blob est de tout savoir de nos désirs et de nos comportements, qui de l'indigène, du bon Brésilien ou bon français, du consommateur, du militant ou du citoyen qui vote. Sachant que l'archi pouvoir/savoir du blob, son droit de tout savoir n'a aucune limite puisqu'il repose sur l'évitement de toute codification par le droit. Cette sortie du droit a été théorisée comme stratégie par Google dès que l'entreprise a rompu avec sa vocation initiale qui était je cite «la mise à disposition universelle, globale et gratuite de l'information », pour vendre les données aux annonceurs et aux boites de conseil. Cette stratégie a pour nom « l'innovation sans permission». Elle est post-libérale et autoritaire comme toute bonne entreprise néolibérale.

Qu'est-ce que ça change pour nous en tant qu'usagers des archives et archivacteurs? Qu'est-ce que qu'on peut y changer? Quelles sont les pratiques et les 


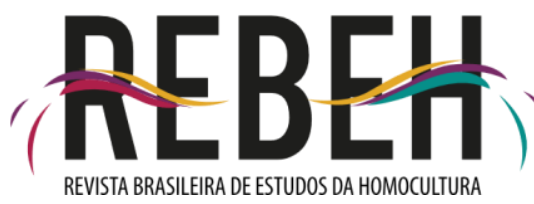

usages de l'archive susceptibles de répondre à la question de savoir quel peut-être le rôle des archives à l'ère néolibérale ?

On pourrait se dire qu'il est utile de rétablir l'histoire et du passé, de la conscience historique ou de l'historicité parce que le néolibéralisme s'en fiche et impose un autre rapport à l'archive avec son blob numérique. Mais peut-être qu'il s'agit d'imposer un refus de ce que fricote le néolibéralisme avec l'histoire et la culture, dont la nôtre.

Il n'est pas exact de dire que le capitalisme néolibéral autoritaire a laissé tomber l'histoire, le récit et la conscience minoritaire. Il en fait juste un usage instrumental parallèlement à son raid sur les cibles biopolitiques et moléculaires, hyperfragmentées, transindividuelles qu'il fait de nous. En fait, avec l'archive vive et le type de réactivation, de remise en circulation qu'elle autorise, il s'agit de contrer la reterritorialisation du devenir minoritaire qu'impose le capitalisme numérique néolibéral. Le simultanéisme du traitement des archives par le blob, le traitement du nous-bio-archive instantané ne doit pas nous faire perdre de vue la façon dont le néolibéralisme mobilise notre histoire, nos subjectivités, la prise de conscience et les émotions, la colère, le ressentiment et la peur par exemple. Non pour réécrire l'histoire mais pour déclencher les guerres culturelles. Et avec succès, si on en juge par les victoires électorales d'un Trump ou d'un Bolsonaro ou le vote du Brexit. Entre autres.

Et il est ironique de voir le rôle joué par les gays et leur expertise en matière de vécu et de rhétorique minoritaire dans les boîtes de traitement des méga données voyous. D'un gay libéral et vegan dingue de mode, ancien directeur de recherches de l'agence en conseil politique Cambridge Analytica comme Christopher Wyle. Wyle a travaillé avec Steve Bannon pour les campagnes de Trump mais aussi d'Obama en 2008 où c'est là qu'il a découvert le microciblage électoral.

D'analyste de data, Wyle s'est transformé en designer de récits efficaces et en promoteur de politiques de l'identité sur le terrain pour gagner la guerre culturelle de Trump. Le mur, le marais, c'est lui. Il a mis sa culture de la politique minoritaire, celle de la politique des identités LGBT, au service de la campagne de Trump. Il en fait une exploitation inversée en jouant sur les ressorts de l'identification et de la victimisation. Le Blanc est victime, il a perdu ses privilèges. Wyle tire des analogies explicatives entre 


\section{员드댄}

la marche des fiertés et les revendications des racistes ou des incels, les célibataires involontaires misogynes et suprématistes, en transposant la rhétorique minoritaire gaie, lesbienne et trans: ils souffrent d'être je cite « au placard ». Ils ressentent le fait de devoir «passer » au sens de «passing » pour les hommes qu'ils ne sont pas ou ne veulent pas être, comme une oppression. C'est tout ça que Wyle a expliqué à Bannon et Trump.

Cette exploitation de la bio-archive numérique et des possibilités qu'elle offre en matière de politique du récit et de subjectivation est assez représentative de ce qui se passe quand se conjuguent plusieurs formes d'exceptionnalisme: l'exceptionnalisme entrepreneurial quand l'état devient néolibéral, quand l'état prend la forme de l'entreprise qui s'empare de la forme de l'état avec Trump, Macron et Bolsonaro, le tout sur fond d'exceptionnalisme raciste.

Si l'exceptionnalisme d'état et l'exceptionnalisme des GAFA, c'est blanc bonnet et bonnet blanc, il n'est pas étonnant que les archives institutionnelles et le blob archival numérique aient en commun une culture du secret, de la non mise à disposition de nos archives et une propension à se mettre hors la loi sur fond d'autoritarisme. Ca revient à faire très exactement le contraire de la mission démocratique affichée qui promet la transparence et la circulation de l'information pour tous, qu'il s'agisse de la mission des Archives nationales depuis la révolution française, des archives nationales du Brésil ou de celle de Google à ses débuts.

Le blob numérique incite au vol et à la dépossession de la même manière que les Archives nationales avec des twists dans la forme. Ces dernières refusent la resynchronisation des producteurs d'archives avec leurs et les archives en installant une temporalité inhumaine avec l'allongement des délais de communicabilité, la qualification changeante des archives, la typologie des lecteurs et des usagers : les pro et les autres, les savants et les amateurs. Le blob numérique, lui, nous refuse la restitution et donc la resynchronisation avec nos traces numériques et leurs sous-produits alors même qu'il nous plonge dans une inscription/exploitation archivale et ineffaçable. C'est de la fausse sursynchronisation si l'on peut dire puisque la vraie source des archives, c'est-à-dire nous, est coupée, ébarbée, séparée et silencée. Le circuit archival est court-circuité et de nouveau la communicabilité entravée. 


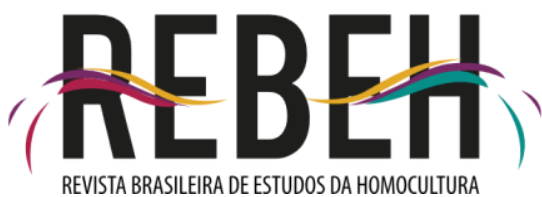

Notre tournant archival à venir, c'est peut-être celui-ci : la re-synchronisation pour toustes qui se traduit par le raccourcissement voir le refus des délais de communication des archives institutionnelles et ... la rémunération de nos clics, la récupération de nos données, de nos bio-data et le refus de leur exploitation. En attendant, c'est le rôle de l'archive vive que de réaliser la resynchronisation dans les centres d'archives autonomes et communautaires.

Je vous remercie...

Recebido em: 10/03/2021

Aceito em: 13/03/2021 